\section{Safety, tolerability, and bioavailability of topical SAR 1118, a novel antagonist of lymphocyte function-associated antigen-1: a phase $1 \mathrm{~b}$ study}

DM Paskowitz', QD Nguyen', P Gehlbach', JT Handa', S Solomon', W Stark', O Shaikh', C Semba', TR Gadek² and DV Do
${ }^{1}$ Wilmer Eye Institute, Johns Hopkins University, Baltimore, MD, USA

2SARcode Corporation, San Francisco, CA, USA

Correspondence: DV Do, Wilmer Eye Institute, Johns Hopkins University, 600 North Wolfe Street, Maumenee 745, Baltimore, MD 21287, USA Tel: +1 410502 0766; Fax: +1 4105021712 . E-mail: ddo@jhmi.edu

Received: 2 November 2011 Accepted in revised form: 20 February 2012 Published online: 27 April 2012

This work was presented at the ARVO Annual Meeting in May 2011.

Daniel Paskowitz is a Fellow of the Heed Ophthalmic Foundation.

\begin{abstract}
Purpose A growing body of evidence points to a role for inflammation mediated by lymphocyte function-associated antigen-1 (LFA-1) and its ligand intercellular adhesion molecule-1 in the pathogenesis of diabetic macular oedema. This phase $1 \mathrm{~b}$ clinical trial assessed the safety, tolerability, and pharmacokinetics of topically administered SAR 1118, a novel LFA-1 antagonist, in human subjects.

Methods In this prospective, randomized, double-masked trial, 13 subjects scheduled for vitrectomy received one of three concentrations of topical SAR $1118(0.1,1.0$, or $5.0 \%$ ) twice daily for 1 week before surgery. Undiluted aqueous and vitreous samples were collected at surgery and analysed for the concentration of the medication.

Results All subjects completed the entire course of medication. The only adverse events reported were instillation site irritation $(4 / 13,31 \%)$ and dysgeusia $(3 / 13$, $23 \%)$. These were mild and transient, occurring at the highest dose. Mean concentrations $(\mathrm{ng} / \mathrm{ml})$ of SAR 1118 in the aqueous humour were $0.25,37.2$, and 101.1 for the $0.1 \%, 1.0 \%$, and $5.0 \%$ dose groups, respectively. SAR 1118 was below the level of detection $(0.5 \mathrm{ng} / \mathrm{ml})$ for all vitreous samples except in a single subject who had a history of prior vitrectomy and a dislocated intraocular lens.

Conclusions Topical SAR 1118 was safe and well tolerated, and dose-dependent
\end{abstract}

levels of drug were detected in aqueous. However, vitreous levels were below the threshold of detection with the concentrations tested. Further investigation of this medication for posterior segment applications would require intravitreal delivery or chemical modification of the drug.

Eye (2012) 26, 944-949; doi:10.1038/eye.2012.68; published online 27 April 2012

Keywords: diabetic retinopathy; macular oedema; intercelluar adhesion molecule-1; pharmacokinetics

\section{Introduction}

Diabetic macular oedema (DME) is a common and vision-threatening manifestation of diabetic retinopathy. Diabetes mellitus affects $\sim 180$ million people worldwide, with the number expected to rise to 300 million by the year $2025 .^{1}$ The population-based Wisconsin Epidemiological Study of Diabetic Retinopathy found that $28 \%$ of patients had DME 20 years after the diagnosis of either type 1 or type 2 diabetes. ${ }^{2}$

Several treatment options are currently available for DME. The Early Treatment Diabetic Retinopathy Study (ETDRS) showed that focal/grid laser photocoagulation reduced the 3-year risk of losing three or more lines of visual acuity by half, from $30 \%$ in the control group to $15 \%$ in the laser-treated group. ${ }^{3}$ More recently, intravitreal vascular endothelial growth factor (VEGF) inhibitors have been 
shown to improve visual acuity and decrease retinal thickening in eyes with DME. ${ }^{4-6}$ However, additional treatment approaches based on our evolving understanding of diabetic retinopathy have the potential to improve visual outcomes further.

A growing body of evidence points to a central role for inflammation in the pathogenesis of DME.,8 Retinal leukostasis, increased capillary permeability, and localized tissue oedema, as seen in DME, are hallmarks of inflammation. Intravitreal injection of triamcinolone, an anti-inflammatory steroid, has shown short-term benefit in the treatment of DME. ${ }^{9}$ Several molecular mediators of inflammation are upregulated in the retina of patients with diabetes or in animal models of diabetic retinopathy. ${ }^{7,8,10}$ Inhibition of some of these mediators has been shown to slow the progression of DME-like features of diabetic retinopathy in animal models. ${ }^{10}$

Strong evidence for the role of inflammation in diabetic retinopathy comes from studies of retinal leukostasis. Leukostasis is mediated by lymphocyte functionassociated antigen-1 (LFA-1; CD11a/CD18; $\alpha$ L $\beta 2$ ), an integrin receptor expressed on leucocytes, and its ligand intercellular adhesion molecule-1 (ICAM-1), expressed on capillary endothelial cells. ICAM- 1 expression is upregulated in the retinal and choroidal vasculature in diabetic patients and diabetic rodent models. ${ }^{11,12}$ ICAM-1 upregulation is accompanied by markedly increased numbers of leucocytes, which cause capillary obstruction, endothelial cell damage, and vascular leakage. ${ }^{13,14}$ Blockade of the LFA-1/ICAM-1 interaction with an injected monoclonal antibody, or by deletion of the gene encoding ICAM-1, reduces leukostasis, vascular leakage, and endothelial cell death, preserving the integrity of the blood-retinal barrier. ${ }^{12,14,15}$

The evidence for a role for LFA- 1 and ICAM- 1 in the pathogenesis of DME suggests that LFA-1 or ICAM-1 antagonists could offer new approaches to the prevention or treatment of DME. SAR 1118 is a novel small-molecule antagonist of the interaction between LFA-1 and ICAM-1. Topically administered SAR 1118 has been shown to reach therapeutic levels in the rat retina, sufficient to reduce leukostasis and blood-retinal barrier breakdown in a diabetic model. ${ }^{16}$ The purpose of this study was to determine the safety, tolerability, and bioavailability of topically administered SAR 1118 in human subjects, as a first step toward evaluating its therapeutic potential for DME.

\section{Materials and methods}

\section{Study participants}

Patients eligible to participate in this study were men or women over 18 years of age scheduled for pars plana vitrectomy for a variety of indications, including epiretinal membrane, vitreomacular traction, vitreous haemorrhage, dislocated intraocular lens (IOL), or intraocular inflammation. Patients already scheduled for vitrectomy for unrelated reasons were chosen for this study because this made it possible to collect vitreous samples without additional surgical risk to the study subjects. The eye scheduled for surgery was the study eye. Women of childbearing potential were included only if they were willing to not become pregnant and to use a reliable form of birth control.

Ocular exclusion criteria for the study eye included active infectious or autoimmune disease of the eye requiring topical medical therapy; glaucoma requiring topical medications; a history of prior vitrectomy or IOL placement within 60 days of study enrolment; history of intravitreal steroids administered to the study eye within 90 days of enrolment or intravitreal VEGF inhibitors within 30 days of enrolment; or use of any topical ophthalmic medication (excluding saline tear lubricants) in the study eye for any reason within 14 days of enrolment. Systemic exclusion criteria included participation in any other studies of investigational drugs or medical devices within 30 days of enrolment; positive pregnancy test; any history of HIV disease or other immunodeficiency disorder; history of acute hepatitis A (IgM positive), hepatitis B, or hepatitis C; history of organ or bone marrow transplant; malignancy under active treatment; or any other acute or chronic medical condition that would, in the judgment of the study investigators, reasonably preclude participation in a clinical study.

\section{Treatment protocol}

Participants were randomized in a 1:1:1 ratio to receive $0.1,1.0$, or $5.0 \%$ SAR 1118 applied topically to the study eye twice per day for 1 week before surgery. The schedule of study visits and evaluations is given in Table 1. A medical and ocular history, general physical examination, screening laboratory tests, and comprehensive eye examination were performed before the first administration of study medication. Participants who met all criteria and who gave informed consent were given a supply of SAR 1118 study medication, but were masked to the dose. Patients were taught to self-administer the medication, and were asked to keep a written record of each drop instilled. SAR 1118 was packaged in sterile unit dose vials in non-preserved phosphate buffered saline at neutral $\mathrm{pH}$; vials were designed to deliver a 50-microliter drop volume.

On the morning of surgery, the final drop was administered by a study investigator $2 \mathrm{~h}$ before the start of the operation. In the operating suite, the study eye was 
Table 1 Screening, treatment, and follow-up protocol

\begin{tabular}{|c|c|c|c|c|c|c|c|}
\hline \multirow[t]{2}{*}{ Assessment } & \multirow[t]{2}{*}{ Screening } & \multicolumn{3}{|c|}{ Treatment period } & \multicolumn{3}{|c|}{ Observation period } \\
\hline & & Do & D1-D7 & D8 (Day of surgery) & D9 (POD \#1) & D10-13 & $D 15 \pm 2$ \\
\hline Informed consent & $x$ & & & & & & \\
\hline Medical history & $X$ & & & & & & \\
\hline Vital signs, height, weight ${ }^{\mathrm{a}}$ & $\mathrm{X}$ & & & & & & $X$ \\
\hline Physical examination & $x$ & & & & & & $x$ \\
\hline Ophthalmic examination ${ }^{\mathrm{b}}$ & $x$ & & & $X$ & $X$ & & $x$ \\
\hline Clinical laboratory tests ${ }^{c}$ & $x$ & & & & & & $x$ \\
\hline Serum pregnancy test/FSH ${ }^{\mathrm{d}}$ & $x$ & & & & & & \\
\hline Aqueous fluid sample & & & & $x^{e}$ & & & \\
\hline Vitreous fluid sample & & & & $X^{e}$ & & & \\
\hline Adverse events & & & $X$ & $x$ & $X$ & $X$ & $x$ \\
\hline Concomitant medications & $x$ & & $x$ & $x$ & $X$ & $x$ & $x$ \\
\hline Instructions on self-administration of study drug & $x$ & & & & & & \\
\hline One drop in study eye BID $^{f}$ & & & $x$ & $x$ & & & \\
\hline
\end{tabular}

${ }^{a}$ Height measured only at screening. ${ }^{b}$ Slit lamp biomicroscopy, dilated funduscopic examination, IOP, BCVA (4 M ETDRS), and OCT. OCT was omitted on Day 9 to avoid postoperative artifacts. ${ }^{\mathrm{c}} \mathrm{CBC}$ with differential, BUN, creatinine. ${ }^{\mathrm{d}}$ Post-menopausal females were required to have serum $\mathrm{FSH} \geq 40 \mathrm{mIU} / \mathrm{ml}$ or to use an approved method of contraception. ${ }^{\mathrm{e}}$ Samples obtained $2 \mathrm{~h}$ after last dose of study medication. ${ }^{\mathrm{f}}$ On Day 8 , subjects received only the morning dose, $2 \mathrm{~h}$ before paracentesis.

anaesthetised, prepared, and draped in the usual sterile fashion for ophthalmic surgery. Before commencing vitrectomy, the retinal surgeon obtained a $50-\mu \mathrm{l}$ or larger sample of aqueous fluid using a 30-Gauge needle attached to a syringe through an anterior chamber paracentesis site. Subsequently, a standard 3-port vitrectomy was established without turning on the infusion line. A $100-\mu$ l or larger sample of undiluted vitreous fluid was obtained using a vitreous cutting device. The aqueous and vitreous samples were stored in sterile polypropylene collection tubes and immediately frozen in a liquid nitrogen bath. The samples were shipped on dry ice to Prevalere Life Sciences (Whitesboro, NY, USA), where liquid chromatography and tandem mass spectroscopy were performed to determine the concentration of SAR 1118 as described. ${ }^{17}$

Subjects were managed post-operatively in the usual manner by their surgeons, and underwent comprehensive eye examinations by study investigators on the first and again on the seventh or eighth postoperative day. These examinations included assessment of best-corrected visual acuity and intraocular pressure, slit lamp and dilated funduscopic examination, and OCT (Table 1).

This study was approved by the Johns Hopkins Medicine Institutional Review Board. All subjects provided written informed consent after explanation of the nature and possible consequences of the study. All study procedures adhered to the tenets of the Declaration of Helsinki. We certify that all applicable institutional and governmental regulations concerning the ethical use of human volunteers were followed during this research.

\section{Results}

\section{Participants and safety outcomes}

Thirteen participants completed the study. Four eyes were randomized to $0.1 \%$ SAR 1118 , four eyes to $1.0 \%$ SAR 1118, and five eyes to 5.0\% SAR 1118 (Table 2). The most common indication for surgery was epiretinal membrane (six participants). Three of the thirteen participants, one in each dosage group, were pseudophakic, while the remainder were phakic. Two participants had undergone prior vitrectomy, one in the $1.0 \%$ group and one in the $5.0 \%$ group. There were no statistically significant differences among the three groups with regard to subject age or phakic status (data not shown).

No participant discontinued the study medication or missed any scheduled dose. Four subjects (31\%) reported transient stinging following administration of the study medication, while three subjects (23\%) reported a metallic taste lasting from 10 to $15 \mathrm{~min}$ following administration. All of these effects were observed with administration of the $5.0 \%$ dose. No other ocular or systemic adverse events were noted. No delays in postoperative healing attributable to the administration of an anti-inflammatory drug were observed.

\section{Intraocular concentration of SAR 1118}

Undiluted aqueous humour samples collected at surgery showed detectable levels of SAR 1118 in the anterior chamber of all patients in the 1.0 and $5.0 \%$ dosage groups in a dose-response fashion (Table 2). Three of the four 
Table 2 Patients, surgical variables, and pharmacological outcomes

\begin{tabular}{lllllcrc}
\hline Patient & Surgical indication & Study eye & Phakic status & Prior PPV & SAR 1118 dose & Aqueous level (ng/ml) & Vitreous level (ng/ml) \\
\hline 1 & ERM & OS & Pseudophakic & No & $0.1 \%$ & $<0.5$ & $<0.5$ \\
2 & Cataract & OS & Phakic & No & $0.1 \%$ & $<0.5$ & $<0.5$ \\
3 & ERM & OS & Phakic & No & $0.1 \%$ & $<0.5$ & $<0.5$ \\
4 & ERM & OD & Phakic & No & $0.1 \%$ & 1.0 & $<0.5$ \\
5 & VMT & OS & Phakic & No & $1.0 \%$ & 1.4 & $<0.5$ \\
6 & Steroid implant & OS & Phakic & No & $1.0 \%$ & 3.0 & $<0.5$ \\
7 & ERM & OS & Pseudophakic & Yes & $1.0 \%$ & 3.2 & $<0.5$ \\
8 & VH & OD & Phakic & No & $1.0 \%$ & 141.0 & $<0.5$ \\
9 & ERM & OD & Phakic & No & $5.0 \%$ & 299.0 & $<0.5$ \\
10 & ERM & OD & Phakic & No & $5.0 \%$ & 117.0 & $<.5$ \\
11 & Dislocated IOL & OS & Dislocated IOL & Yes & $5.0 \%$ & 14.9 & $<.1$ \\
12 & Diagnostic PPV & OS & Phakic & No & $5.0 \%$ & 52.0 & $<0.5$ \\
13 & VH & OS & Phakic & No & $5.0 \%$ & 22.6 & $<0.5$ \\
\hline
\end{tabular}

Abbreviations: ERM, epiretinal membrane; IOL, intraocular lens; OD, right eye; OS, left eye; PPV, pars plana vitrectomy; VMT, vitreomacular traction.

participants receiving the lowest dose, $0.1 \%$ SAR 1118, had undetectable aqueous levels, while the fourth had a level of only $1.0 \mathrm{ng} / \mathrm{ml}$. Aqueous concentrations of the study drug varied widely among the five participants receiving the 1.0 and $5.0 \%$ doses, with higher average levels in the $5.0 \%$ group.

All participants in the 0.1 and $1.0 \%$ groups had undetectable vitreous levels of SAR 1118. In the highest dose group (5.0\%), four out of five participants had undetectable vitreous levels of SAR118. One participant had a vitreous concentration of $8.1 \mathrm{ng} / \mathrm{ml}$ (Table 2). This participant had a history of both pseudophakia with dislocated IOL and prior vitrectomy in the study eye.

\section{Discussion}

This study was motivated by the lack of an effective topical medical therapy for DME. Several lines of preclinical evidence have implicated inflammation mediated by LFA-1 and ICAM-1 in the pathogenesis of DME, suggesting that the LFA-1 antagonist SAR 1118 might offer a novel treatment for this common and vision-threatening complication of diabetes. In this Phase $1 b$ randomized clinical trial, topical SAR 1118 was well tolerated and was not associated with any severe ocular or systemic adverse events. In vitro analyses have determined that SAR 1118 effectively inhibits the LFA-1/ICAM-1 interaction at concentrations of $30 \mathrm{ng} / \mathrm{ml}$ or greater (data not shown). In our subjects receiving the $5.0 \%$ dose, topical SAR 1118 achieved pharmacologically relevant levels in the aqueous humour. Ongoing studies are addressing the possible value of this drug in the treatment of anterior segment inflammatory syndromes, including dry eye, anterior uveitis, and postoperative inflammation.

Interestingly, a high aqueous level of drug was noted in a single patient who received the $1.0 \%$ dose (patient 8; see Table 2), well above the consistently low levels found in the other three patients receiving this dose. This patient's indication for vitrectomy was vitreous haemorrhage secondary to proliferative diabetic retinopathy. However, the vitreous haemorrhage alone is unlikely to account for the elevated aqueous level of SAR 1118 , because patient 13 , who also had a vitreous haemorrhage related to proliferative disease, was also phakic, and received a higher dose of the study medication, had a much lower aqueous level (Table 2). Timing of drug administration is also unlikely to explain the finding. The final dose of study medication was administered by an investigator $2 \mathrm{~h}$ before the start of surgery for each patient. While the exact timing of the final eyedrop relative to sample collection varied by a few minutes from patient to patient, this would not be sufficient to explain a difference of two orders of magnitude in the aqueous level. The most likely explanation for the increased aqueous level in patient 8 is altered aqueous humour dynamics. This patient with proliferative diabetic retinopathy may have had subtle neovascularization of the angle, leakage of vitreous blood into the anterior chamber, or both. These factors would be expected to impair aqueous drainage via the trabecular meshwork and to markedly increase accumulation of the study drug in the anterior chamber.

SAR 1118 was not detected in the vitreous except for a single participant who received the highest dose and had a history of prior vitrectomy and cataract surgery with a dislocated IOL. This subject had a much lower aqueous level compared with the other three patients in the $5.0 \%$ group, arguing that the relatively high vitreous level was not a result of inadvertent administration of extra drops or of a shorter interval between the final drop and sample collection, which would also have elevated the aqueous level. Instead, the higher vitreous penetration in this 
patient may have been related to the posteriorly dislocated IOL (the indication for vitrectomy in this case), creating single-chamber pharmacokinetics that enhanced the posterior segment level of SAR 1118. Furthermore, the prior vitrectomy had removed the hyaloid face, further enhancing the free circulation of fluid from the anterior chamber into the posterior segment. These results suggest that an intact native lens or an intact hyaloid can serve as a barrier to diffusion of SAR 1118 into the posterior segment of the human eye. Prior studies in rats have shown excellent retinal bioavailability of topically administered SAR $1118 .{ }^{16}$ The larger size of the human eye, thicker ocular coats, differences in the composition of the hyaloid, or other unknown factors may account for the different pharmacokinetics of SAR 1118 in the human eye compared with the rat.

The treatment of pseudophakic cystoid macular oedema with topical non-steroidal anti-inflammatory medications offers an example of successful topical treatment of a macular disorder in the presence of an intact hyaloid. ${ }^{18,19}$ It may be possible to chemically modify SAR 1118 to improve its posterior segment penetration when delivered topically. A recent report of successful reduction of uveitic macular oedema using subcutaneous injection of efalizumab, a humanized antibody directed against a subunit of LFA- $1,{ }^{20}$ highlights the therapeutic potential of therapies designed to inhibit LFA-1/ICAM-1. Further research is necessary to determine whether LFA-1/ICAM-1 inhibitors have a role in the treatment of DME.

\section{Summary}

What was known before

- Diabetic macular oedema is a common and serious consequence of diabetic eye disease.

- No topical medical treatment is available for diabetic macular oedema.

- Considerable evidence suggests that intercellular adhesion molecule-1 (ICAM-1) has an important role in the pathogenesis of diabetic macular oedema.

What this study adds

- The ICAM-1 antagonist SAR 1118 is safe and well tolerated when applied topically to the human eye.

- SAR 1118 has favourable pharmacokinetics for therapeutic use in the anterior segment of the human eye.

\section{Conflict of interest}

Charles Semba and Thomas R Gadek are employees of SARcode Corporation. The remaining authors declare no conflict of interest.

\section{References}

1 King H, Aubert RE, Herman WH. Global burden of diabetes, 1995-2025: prevalence, numerical estimates, and projections. Diabetes Care 1998; 21: 1414-1431.

2 Klein R, Klein BE, Moss SE, Davis MD, DeMets DL. The Wisconsin epidemiologic study of diabetic retinopathy. IV. Diabetic macular edema. Ophthalmology 1984; 91: 1464-1474.

3 Photocoagulation for diabetic macular edema. Early Treatment Diabetic Retinopathy Study report number 1. Early Treatment Diabetic Retinopathy Study research group. Arch Ophthalmol 1985; 103: 1796-1806.

4 Diabetic Retinopathy Clinical Research Network. Randomized trial evaluating ranibizumab plus prompt or deferred laser or triamcinolone plus prompt laser for diabetic macular edema. Ophthalmology 2010; 117: 1064-1077.

5 Scott IU, Edwards AR, Beck RW, Bressler NM, Chan CK, Elman MJ et al. A phase II randomized clinical trial of intravitreal bevacizumab for diabetic macular edema. Ophthalmology 2007; 114: 1860-1867.

6 Cunningham ET Jr, Adamis AP, Altaweel M, Aiello LP, Bressler NM, D'Amico DJ et al. A phase II randomized double-masked trial of pegaptanib, an anti-vascular endothelial growth factor aptamer, for diabetic macular edema. Ophthalmology 2005; 112: 1747-1757.

7 Adamis AP, Berman AJ. Immunological mechanisms in the pathogenesis of diabetic retinopathy. Semin Immunopathol 2008; 30: 65-84.

8 Adamis AP. Is diabetic retinopathy an inflammatory disease? Br J Ophthalmol 2002; 86: 363-365.

9 Martidis A, Duker JS, Greenberg PB, Rogers AH, Puliafito $\mathrm{CA}$, Reichel $\mathrm{E}$ et al. Intravitreal triamcinolone for refractory diabetic macular edema. Ophthalmology 2002; 109: 920-927.

10 Kern TS. Contributions of inflammatory processes to the development of the early stages of diabetic retinopathy. Exp Diabetes Res 2007; 2007: 95103.

11 McLeod DS, Lefer DJ, Merges C, Lutty GA. Enhanced expression of intracellular adhesion molecule- 1 and P-selectin in the diabetic human retina and choroid. Am J Pathol 1995; 147: 642-653.

12 Miyamoto K, Khosrof S, Bursell SE, Rohan R, Murata T, Clermont AC et al. Prevention of leukostasis and vascular leakage in streptozotocin-induced diabetic retinopathy via intercellular adhesion molecule-1 inhibition. Proc Natl Acad Sci USA 1999; 96: 10836-10841.

13 Schroder S, Palinski W, Schmid-Schonbein GW. Activated monocytes and granulocytes, capillary nonperfusion, and neovascularization in diabetic retinopathy. Am J Pathol 1991; 139: 81-100.

14 Joussen AM, Murata T, Tsujikawa A, Kirchhof B, Bursell SE, Adamis AP. Leukocyte-mediated endothelial cell injury and death in the diabetic retina. Am J Pathol 2001; 158: 147-152.

15 Joussen AM, Poulaki V, Le ML, Koizumi K, Esser C, Janicki $\mathrm{H}$ et al. A central role for inflammation in the pathogenesis of diabetic retinopathy. Faseb J 2004; 18: 1450-1452.

16 Rao VR, Prescott E, Shelke NB, Trivedi R, Thomas P, Struble $\mathrm{C}$ et al. Delivery of SAR 1118 to the retina via ophthalmic drops and its effectiveness in a rat streptozotocin (STZ) model of diabetic retinopathy (DR). Invest Ophthalmol Vis Sci 2010; 51: 5198-5204.

17 Murphy CJ, Bentley E, Miller PE, McIntyre K, Leatherberry $\mathrm{G}$, Dubielzig R et al. The pharmacological assessment of 
a novel lymphocyte function-associated antigen-1 antagonist (SAR 1118) for the treatment of keratoconjunctivitis sicca in dogs. Invest Ophthalmol Vis Sci 2011; 52: 3174-3180.

18 Flach AJ, Jampol LM, Weinberg D, Kraff MC, Yannuzzi LA, Campo RV et al. Improvement in visual acuity in chronic aphakic and pseudophakic cystoid macular edema after treatment with topical $0.5 \%$ ketorolac tromethamine. Am J Ophthalmol 1991; 112: 514-519.
19 Flach AJ, Dolan BJ, Irvine AR. Effectiveness of ketorolac tromethamine $0.5 \%$ ophthalmic solution for chronic aphakic and pseudophakic cystoid macular edema. Am J Ophthalmol 1987; 103: 479-486.

20 Faia LJ, Sen HN, Li Z, Yeh S, Wroblewski KJ, Nussenblatt RB. Treatment of inflammatory macular oedema with humanized anti-CD11a antibody therapy. Invest Ophthalmol Vis Sci 2011; 52(9): 6919-6924. 